\title{
MODELING THE L(+) LACTIC ACID PRODUCTION VIA R. ORYZAE NRRL 395 FERMENTATION ON BIODIESEL CRUDE GLYCEROL
}

\author{
EVA-H. DULF ${ }^{\mathrm{a}}$, DAN C. VODNAR ${ }^{\mathrm{b}}$, FRANCISC V. DULF ${ }^{\mathrm{b} *}$
}

\begin{abstract}
Similar to the petroleum industry, biodiesel industry generates unwanted by-products. Biodiesel production generate huge amount of crude glycerol- one part of glycerol is produced at every ten parts of biodiesel, and has a negative influence on the biodiesel price. A solution to reduce the negative environmental problems and the cost of biodiesel is to use crude glycerol as carbon source for microbial growth media in order to produce valuable organic chemicals. Recently, the research group from University of Agricultural Sciences and Veterinary Medicine Cluj-Napoca developed sustainable opportunities for $\mathrm{L}(+)$ lactic acid production via R. oryzae NRRL 395 fermentation on biodiesel crude glycerol. The current research aims at improving the production of L-lactic acid using crude glycerol as substrate, immobilized spores and inhibited alcohol dehydrogenase of R. oryzae in discontinuous and semi continuous fermentation processes in order to make the process more efficient. According to several experimental dataset under different process parameters, multiple regression methods were employed to establish mathematical models for prediction of the $L$ lactic acid production. Determination coefficient values states that predicted values were in good agreement with the experimental values. Introducing the fractional order in models lead to a good phenomenological description. The resulted models can be used both in optimization process and prediction instead of time and resource consuming experiments.
\end{abstract}

Keywords: biodiesel; crude glycerol; process optimization, mathematical model, fractional order model

a Technical University of Cluj-Napoca, Faculty of Automation and Computer Science, 28 Memorandumului str., RO-400014, Cluj-Napoca, Romania

b University of Agricultural Sciences and Veterinary Medicine Cluj-Napoca, Faculty of Food Science and Technology, 3-5 Manastur str., RO- 400372, Cluj-Napoca, Romania

*Corresponding author: francisc_dulf@usamvcluj.ro 


\section{INTRODUCTION}

Nowadays, having the authority's pessimistic prospects for the future regarding the absolute dependency on fossil fuels, the political and economic policies are to stimulate the development of sustainable alternatives to fossil fuels. Biodiesel represent an alternative for the substitution of fossil fuels, which is the most common biofuel in Europe [1]. Similar to the petroleum industry, biodiesel industry generate unwanted by-products [2]. Biodiesel production generate huge amount of crude glycerol- one part of glycerol is produced at every ten parts of biodiesel [3], and has a negative influence on the biodiesel price.

The glycerine phase from biodiesel industry causes environmental problems regarding to the management of this waste. A solution to reduce the negative environmental problems and the cost of biodiesel is to use crude glycerol as carbon source for microbial growth media in order to produce valuable organic chemicals [4-6].

$\mathrm{L}(+)$-lactic acid is an important chemical which has applications in many industries: food, cosmetics, pharmaceuticals; etc. It can be used as a feedstock for the production of poly-lactic acid (PLA), a polymer present in medical applications and environmentally friendly biodegradable plastics with high socio-economic impact, which can be substituted for synthetic plastics derived from petroleum resources $[7,8]$.

It is obvious that the optimization of lactic acid fermentation is a highly discussed problem. Still, the problem is not solved from many points of view, and the substrate plays a vital role in the improvement of the process. Raw materials can reduce the cost of substrates. However, pre-treatment cost and low productivity in fermentation using raw materials are the remaining drawbacks to be solved.

More recently, the research team from University of Agricultural Sciences and Veterinary Medicine Cluj-Napoca developed and analysed sustainable opportunities for $\mathrm{L}(+)$ lactic acid production via R. oryzae NRRL 395 fermentation on biodiesel crude glycerol $[9,10]$. Apart from the large group of bacteria, filamentous fungi, especially $R$. oryzae proved that is a good lactic acid producer. Importantly, the filamentous fungus R. oryzae can produce an optically pure $\mathrm{L}(+)$-lactic acid from media without expenses pre-treatment costs. $R$. oryzae NRRL 395 can utilize crude glycerol as carbon source, and unlike its competitors of lactic acid producing bacteria tolerate high impurities, has lower nutrition requirements which reduce the fermentation cost and simplifies downstream product separation.

The current research of the authors aim at improving the production of L-lactic acid using crude glycerol as substrate, immobilized spores and inhibited alcohol dehydrogenase of R. oryzae in discontinuous and semi-continuous 
fermentation processes in order to make the process more efficient. Rigorous mathematical models would be useful both in prediction and in the optimization stage. To the best of our knowledge, there are no such models available for the studied processes. The paper presents the developed mathematical models, highlighting the advantages and disadvantages of each model.

\section{MATERIALS AND METHODS}

The aim of the current paper is to mathematically describe the potentialities of valorisation of crude glycerol by pelletized R. oryzae NRRL 395 , in order to analyse and optimize the production of $L(+)$-lactic acid in media supplemented with laboratory nutrients or with lucerne green juice (LGJ). The model of the $L$ lactic acid production process was developed in Matlab®, based on the data published by the authors in [9]. In this investigation, the input or decision variables consist of the amount of inorganic nutrients and time. As mathematical tool is used the multiple regression, both in linear or nonlinear form [11, 12]. Are also developed fractional order models, being recognized for the advantages introduced in modeling the complexity of the dynamics of some real-world systems $[13,14]$.

\section{RESULTS AND DISCUSSION}

The first developed model describes the dependency of the crude glycerol concentration for cultivating R. oryzae NRRL 395 in trials without LGJ addition and in trial with LGJ.

Noting with $x_{1}$ and $x_{2}$ the crude glycerol (CG) contents supplemented with different amount of inorganic nutrients (IN) and time, respectively, the mathematical model of productivity, $y$, resulted by the multiple linear regression is:

$$
y=-1.1914+0.1571 x_{1}-0.4286 x_{2}+0.0806 x_{1} x_{2}
$$

The corresponding simulation results, for values of $x_{1}$ in the range $10-80$ $\mathrm{g} / \mathrm{L}$ and $\mathrm{x}_{2}$ in the range $20-90 \mathrm{~g} / \mathrm{L}$ are presented in Figure 1 , in comparison with the experimental data (blue dots). The resulted statistical data are: $R_{2}=0.8416$ and $p$-value $=3.41 \mathrm{e}-08$. 


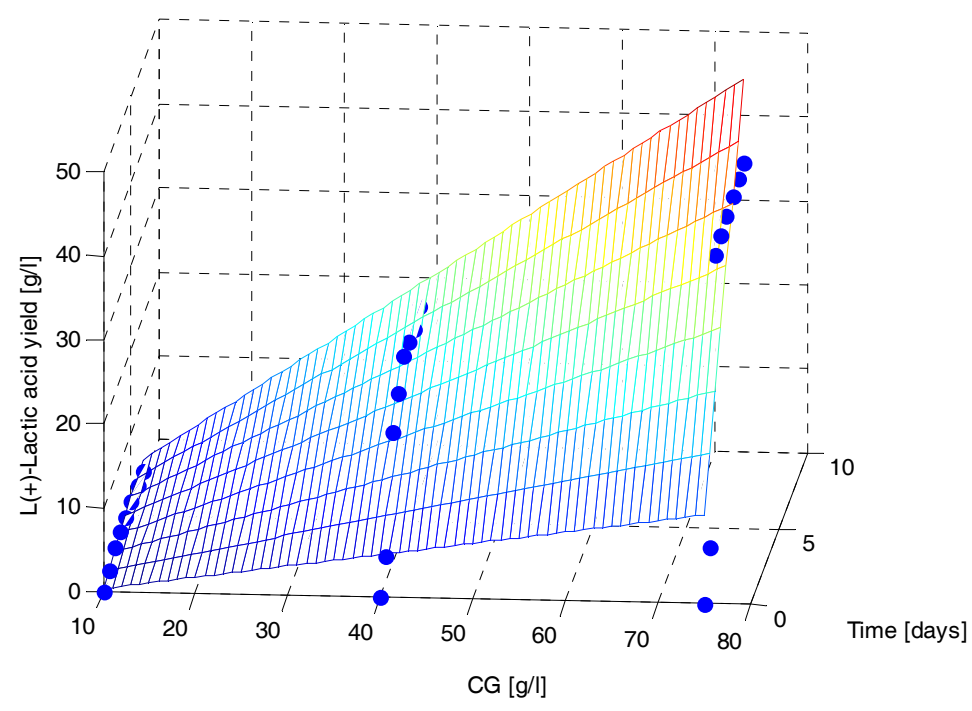

Figure 1. Simulation result with the linear model of $x_{1}, x_{2}$

For a better process description is developed also a nonlinear model, including the quadratic terms:

$$
\begin{aligned}
& y=-8.3012+0.2280 x_{1}+5.7439 x_{2}+0.0806 x_{1} x_{2}- \\
& -0.0008 x_{1}^{2}-0.8818 x_{2}^{2}
\end{aligned}
$$

The corresponding simulation result is in Figure. 2 for the same range of parameters $x_{1}$ and $x_{2}$, highlighting o good match, with $R_{2}=0.9092$ and $p$ value $=9.081 \mathrm{e}-09$.

It has been shown that fractional calculus in general and fractional order differ-integrals models in particular can characterize very well the phenomenology of biochemical processes [15]. Another advantage of these equations is that is simple to derive further a transfer function model from in its Laplace equivalent. The great deal of success is due to the fact that explains complex phenomena with a startling simplicity [16]. 


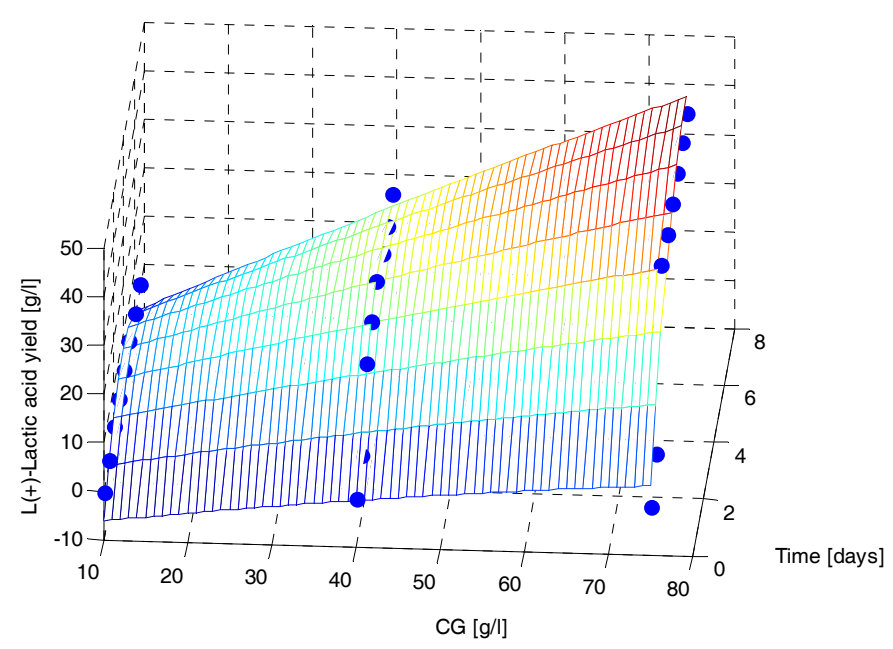

Figure 2. Simulation result with the classical nonlinear model of $x_{1}, x_{2}$

Having this in mind it were developed a series of fractional order models. The best results were obtained for the fractional order model:

$$
\begin{aligned}
& y=-7.9601+0.14646 x_{1}^{1.2}+7.8152 x_{2}^{1.2}+0.0806 x_{1}^{1.7}- \\
& -3.0646 x_{2}^{1.7}
\end{aligned}
$$

with $R_{2}=0.9101$ and $p=8.35 \mathrm{e}-09$.

The graphical plot from Figure 3 reveals a great phenomenological fit.

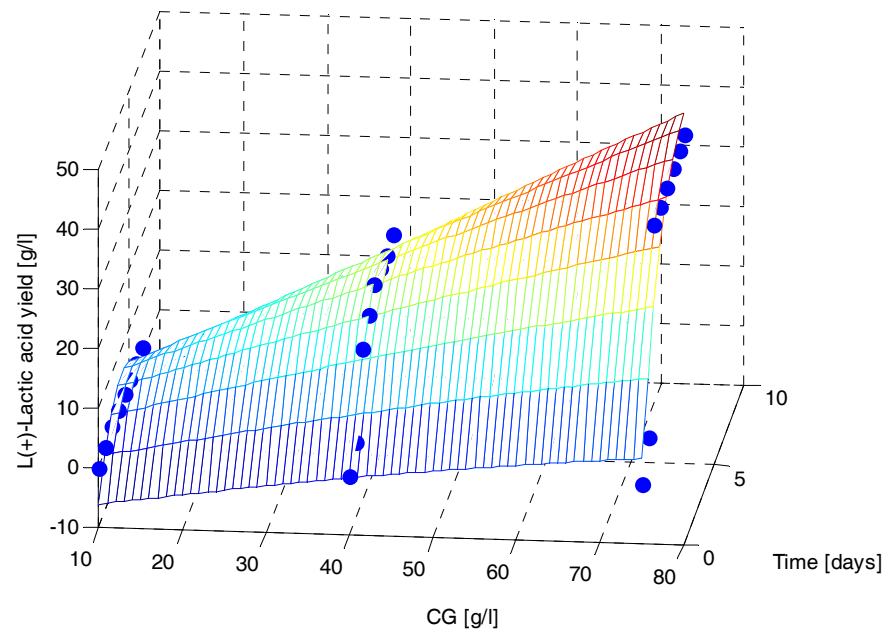

Figure 3. Simulation result with the fractional order model of $x_{1}, x_{2}$ 
In the same manner were developed models including the $L(+)$-lactic acid yield during R.oryzae NRRL 395 fermentaion in media containing different ratio of crude glycerol (CG) $(10,40,75 \mathrm{~g} / \mathrm{l})$ supplemented with lucerne green juice (LGJ) $(90,60,25 \mathrm{~g} / \mathrm{l})$, noted with variable $\times 1$ and $\times 3$. The linear model is:

$$
y=18.5414-0.1936 x_{1}+9.0227 x_{3}-0.0931 x_{1} x_{3}
$$

The nonlinear model becomes:

$$
\begin{aligned}
& y=4.3191+0.0679 x_{1}+17.4021 x_{3}-0.0931 x_{1} x_{3}- \\
& -0.0023 x_{1}^{2}-1.1971 x_{3}^{2}
\end{aligned}
$$

while the fractional order model is:

$$
\begin{aligned}
& y=2.5961+0.1699 x_{1}^{0.85}+19.6730 x_{3}^{0.85}-0.0925 x_{1} x_{3}- \\
& -0.0038 x_{1}^{1.9}-0.9404 x_{3}^{1.9}
\end{aligned}
$$

The corresponding simulation results are presented in Figure 4, Figure 5 and Figure 6. The corresponding statistical measures are: $R_{2}=[0.8077,0.8950$, $0.8936]$ and $p=[2.32 \mathrm{e}-073.28 \mathrm{e}-083.72 \mathrm{e}-08]$, highlighting once again the advantage of fractional order.

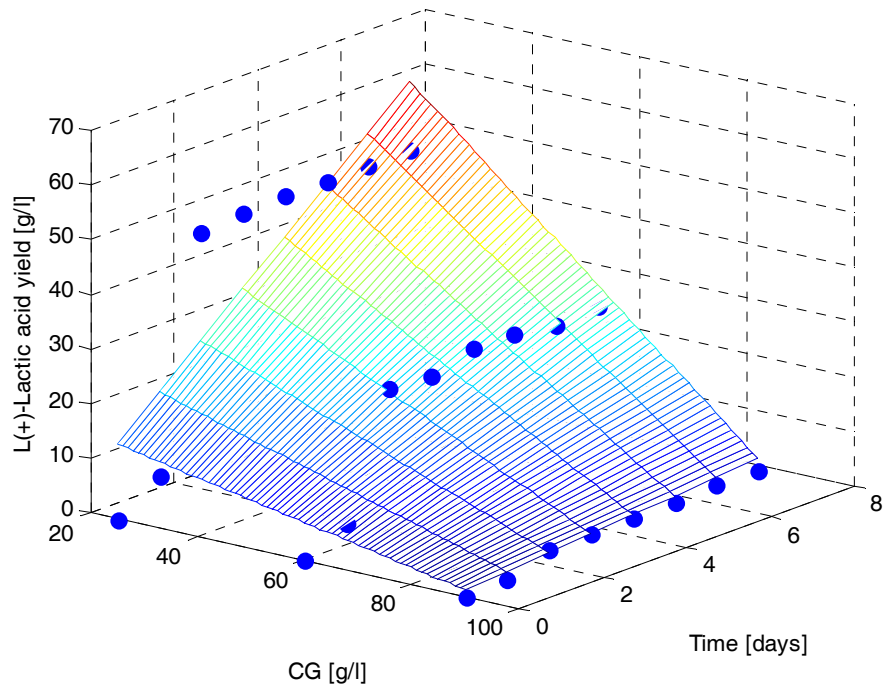

Figure 4. Simulation result with the linear model of $x_{1}, x_{3}$ 


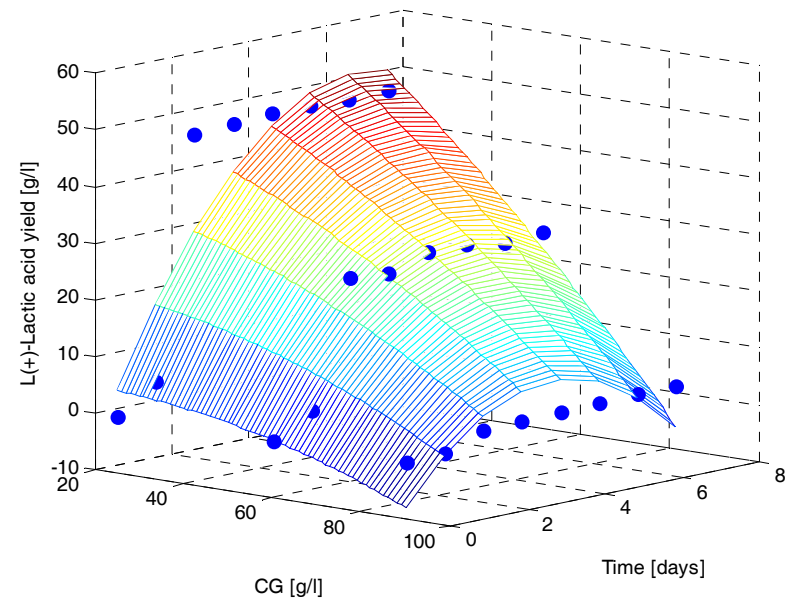

Figure 5. Simulation result with the classical nonlinear model of $x_{1}, x_{3}$

The mathematical model containing all three parameters is:

$$
\begin{aligned}
& y=-116.3715 x_{2}+1.3855 x_{1} x_{2}+0.8672 x_{1} x_{3}+0.7246 x_{2} x_{3}- \\
& -0.1050 x_{1} x_{2} x_{3}+0.0113 x_{1}^{2}+1.1389 x_{2}^{2}+6.7875 x_{3}^{2}
\end{aligned}
$$

with a mean square error of $3.6274 \cdot 10^{-13}$.

In this case is also obtained the minimum error for the fractional order model: $6.8359 \cdot 10^{-14}$, having the form:

$$
\begin{aligned}
& y=-4.5443 x_{2}+0.0306 x_{1} x_{2}-0.0153 x_{1} x_{3}+ \\
& +0.0115 x_{2} x_{3}-0.0032 x_{1} x_{2} x_{3}+0.0258 x_{1}^{1.8}+0.4457 x_{2}^{1.8}+ \\
& +1.7604 x_{3}^{1.8}
\end{aligned}
$$

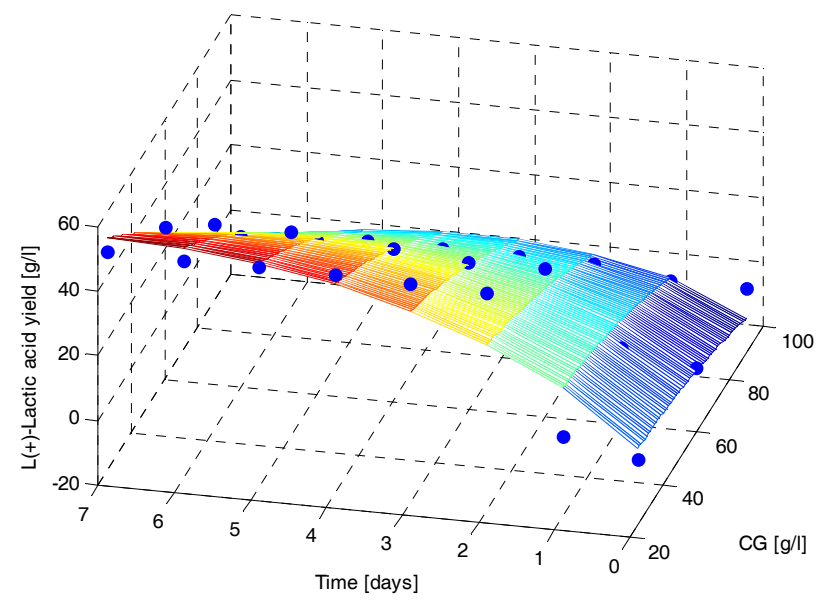

Figure 6. Simulation result with the fractional order model of $x_{1}, x_{3}$ 
The kinetics study of lactic acid production in bioreactor consisted in the analysis of the capacity of R. oryzae NRRL 395 for sugar utilization as model media. Processing the experimental results of R. oryzae NRRL 395 in producing $L(+)$-lactic with classical identification method, namely the Instrument Variable approach, lead to the integer order model in Laplace form:

$$
H(s)=\frac{60}{(0.3 s+1)(0.65 s+1)}
$$

where the system input is the ratio of crude glycerol (CG) and the output is the $L(+)$-lactic yield. The resulted mean square error is of 1.54 . The corresponding fractional order model, identified as in $[13,14]$ is:

$$
H(s)=\frac{60}{\left(0.3 s^{1.2}+1\right)\left(0.65 s^{0.8}+1\right)}
$$

with a mean square error of 0.89 . The comparative plot resulted with these two models is presented in Figure 7.

The linear mathematical model describing the R. oryzae NRRL 395 for sugar utilization for different ratio of crude glycerol (CG) resulted as:

$$
H(s)=\frac{100}{(0.4 s+1)(0.8 s+1)}
$$

with a mean square error of 3.27. The fractional order model has the form:

$$
H(s)=\frac{100}{\left(0.4 s^{1.3}+1\right)\left(0.8 s^{0.8}+1\right)}
$$

and the corresponding error is less than 0.45 . The comparative simulation results of integer order and fractional order models are presented in Figure 8.

The next analysis consists on the lactic acid production by R. oryzae NRRL 395 on crude glycerol supplemented with inorganic nutrients. The results highlight once again the superiority of the fractional order models, Figure 9 . The obtained values are in agreement with the data reported in the specific literature. 


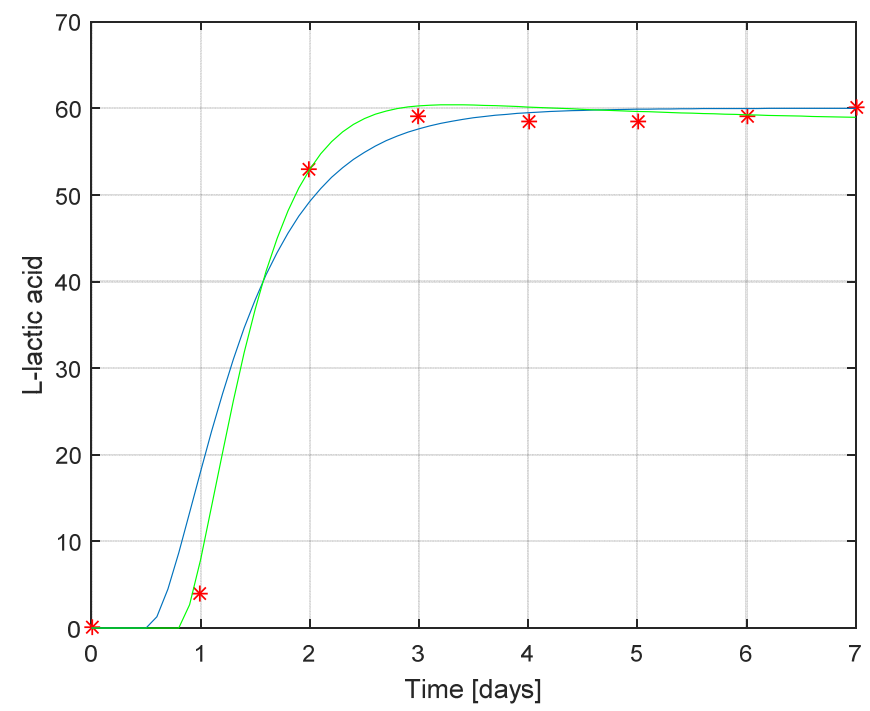

Figure 7. Simulation results for an integer order and fractional order model in producing $L(+)$-lactic (red stars-experimental data, blue line - linear model, green line - fractional order model)

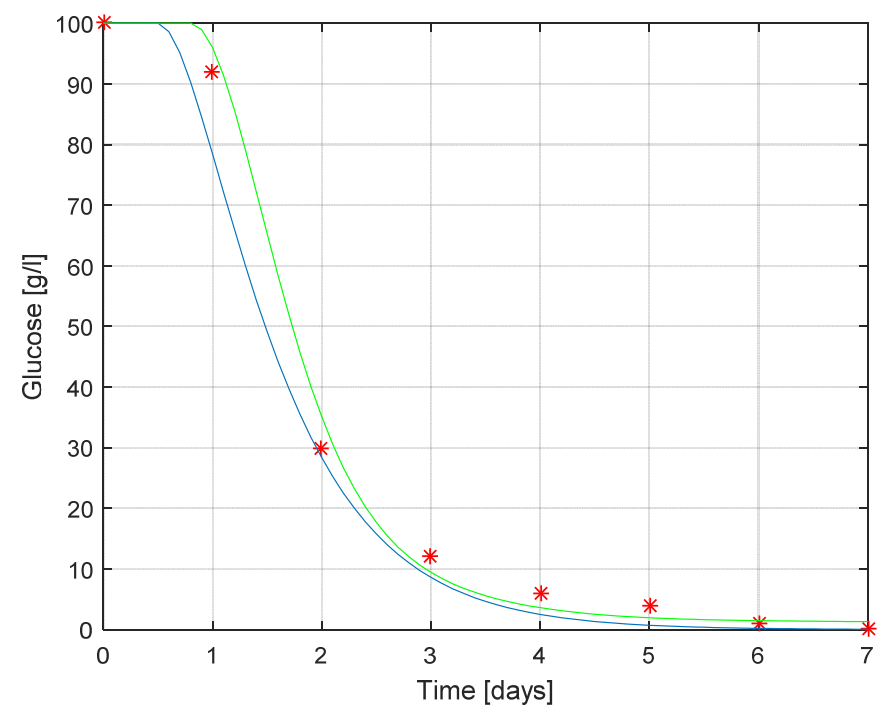

Figure 8. Simulation results for an integer order and fractional order model in sugar utilization (red stars-experimental data, blue line - linear model, green line - fractional order model) 


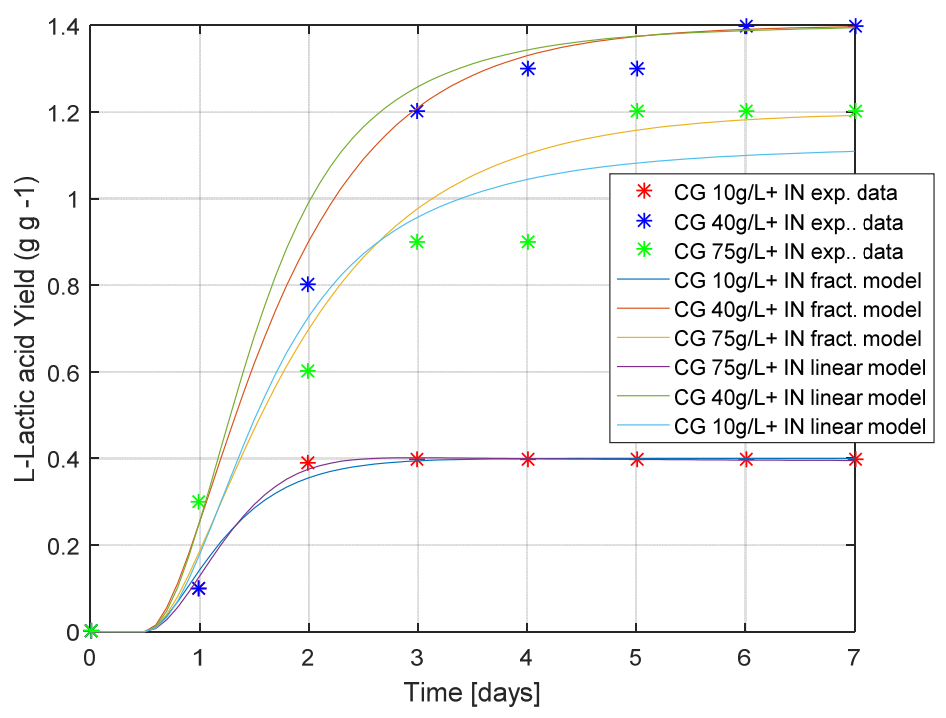

Figure 9. Simulation results for $\mathrm{L}(+)$-lactic acid production obtained in trials with crude glycerol (CG) $(10,40$, and75 g l-1)

\section{CONCLUSIONS}

The aim of the current paper was to mathematically describe the phenomena in the $\mathrm{L}(+)$ lactic acid production via $R$. oryzae NRRL 395 fermentation on biodiesel crude glycerol, in order to analyze and optimize the production in media supplemented with laboratory nutrients or with Lucerne green juice (LGJ). From the obtained mathematical models the best results are provided with the fractional order models, describing very well the nonlinear phenomena and being easy to use for optimization. The obtained mean square error is $6.8359 \cdot 10^{-14}$. The kinetic study of lactic acid production leads to a mean square error of 0.45 with the fractional order model, while with the integer order model is 3.27 .

Future works include the application of different optimization methods for these processes.

\section{ACKNOWLEDGMENTS}

This work was supported by a grant of the Romanian National Authority for Scientific Research, CNDI- UEFISCDI, project number PN-III-P2-2.1-PED-20161237, contract 17PED/2017 and project PN-III-P1-1.2-PCCDI2017-0056 contract 2PCCDI/2018. 
MODELING THE L(+) LACTIC ACID PRODUCTION VIA R. ORYZAE NRRL 395 FERMENTATION ON BIODIESEL CRUDE GLYCEROL

\section{REFERENCES}

1. A. Drozdzynska, K. Leja, K. Czaczyk, J Biotechnol Comp Bio Bionanotechnol., 2011, 92, 92.

2. R. Dobson, V. Gray, K. Rumbold, J Ind Microbiol Biotechnol., 2012, 39, 217.

3. D.M. Rossi, J.B. da Costa, E.A. de Souza, M.C.R. Perelba., M.A.Z. Ayub, Renew Energ, 2012, 39, 223.

4. A. Kosmider, A. Drozdzynska, K. Blaszka, K. Leja, K. Czaczyk, Pol J Environ Stud., 2010, 19, 1249.

5. F. Yang, M.A. Hanna, R. Sun,Biotechnol Biofuel., 2012, 5(13), 1.

6. K. Xu, P. Xu, Bioresour Technol., 2014, 153, 23.

7. M.A. Abdel-Rahman, Y.Tashiro, K. Sonomoto, Biotechnol Adv., 2013, 31, 877.

8. Y. Wang, Y. Tashiro, K. Sonomoto, J Biosci Bioeng, 2014, available online.

9. D.C. Vodnar, F.V. Dulf, O.L. Pop, C. Socaciu, Microb Cell Fact., 2013, 12, 92.

10. D.C. Vodnar, L.F. Calinoiu, F.V. Dulf, B.E. Stefanescu, G. Crisan, C. Socaciu, Food Chemistry, 2017, 231, 131.

11. D. Olive, "Linear Regression", Springer Verlag, 2017.

12. B.R. Darlington, A.F. Hayes, "Regression Analysis and Linear Models Concepts, Applications, and Implementation", The Guilford Press, 2016.

13. J.A.T Machado;V. Kiryakova, F. Mainardi, Communications in Nonlinear Science and Numerical Simulation, 2011, 16(3), 1140.

14. C.A. Monje, Y.Q.Chen, B.M. Vinagre, D. Xue, V. Feliu, "Fractional-order Systems and Controls", Springer Verlag, 2010, chapter 4.

15. E.H Dulf, F.V. Dulf, C.I. Pop, Chemical Engineering Communication, 2015, 202(12), 1600.

16. E.H. Dulf, C.I. Muresan, M.L. Unguresan, Journal of Mathematical Chemistry, 2014, 52(1), 115. 
